Supplement of Geochronology, 3, 383-394, 2021

https://doi.org/10.5194/gchron-3-383-2021-supplement

(c) Author(s) 2021. CC BY 4.0 License.

(c) (i)

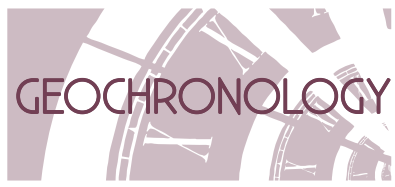

Supplement of

\title{
AI-Track-tive: open-source software for automated recognition and counting of surface semi-tracks using computer vision (artificial intelligence)
}

Simon Nachtergaele and Johan De Grave

Correspondence to: Simon Nachtergaele (simon.nachtergaele@ugent.be)

The copyright of individual parts of the supplement might differ from the article licence. 


\section{Al-Track-tive offline}

* How to install?

- download AI-Track-tive project on

https://github.com/SimonNachtergaele

- follow instructions for Windows, Mac-OS or Linux

- open .exe file or run Python code in Python code editor

* Determines track density in apatite/mica (tutorial available)

- load .weights file (DNN for apatite) from option A or B

- load weights file (DNN for mica) obtained in option A or B

- load Yolov3 configuration file (.cfg)

- select reflected light and transmitted light images of apatite and/or ED

- select Region Of Interest: square, custom polygon or circle

- manual check of the automatic track identification results

* Live fission track recognition (tutorial available)

* Etch pit diameter (Dpar) measurement (tutorial available)
Option A: Use our Deep Neural Networks

- free to download on

https://github.com/SimonNachtergaele

Option B: Make your own Deep Neural Network

* Create training dataset using Labelimg

- download Labelimg on

https://github.com/tzutalin/labellmg

- annotate tracks in 50 apatite images

- annotate tracks in 50 external detector images

* Execute Jupyter notebook in Google Colab

- download Jupyter notebook (.ipynb) on

https://github.com/SimonNachtergaele

- connect to Google Colab

- iterate

- download weights file from Google Drive

\section{Graphical User Interface (GUI)}

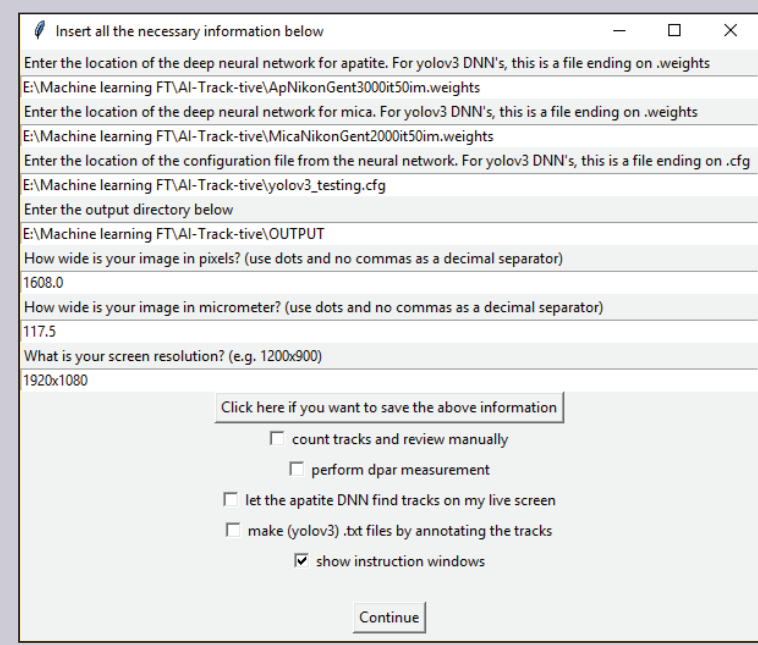

Insert DNN and other info

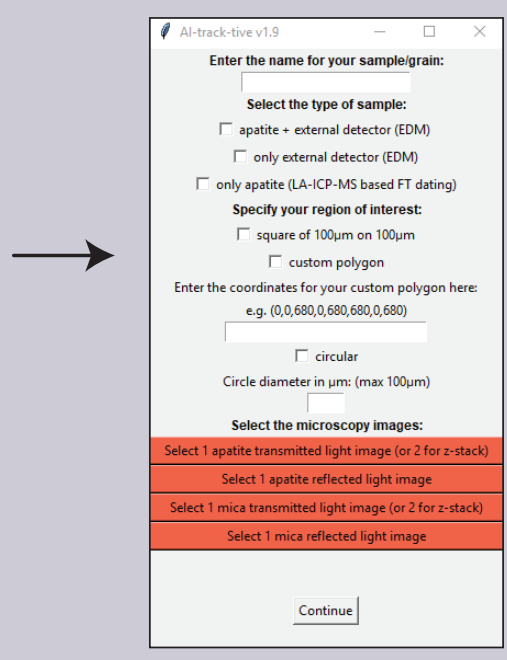

Select images

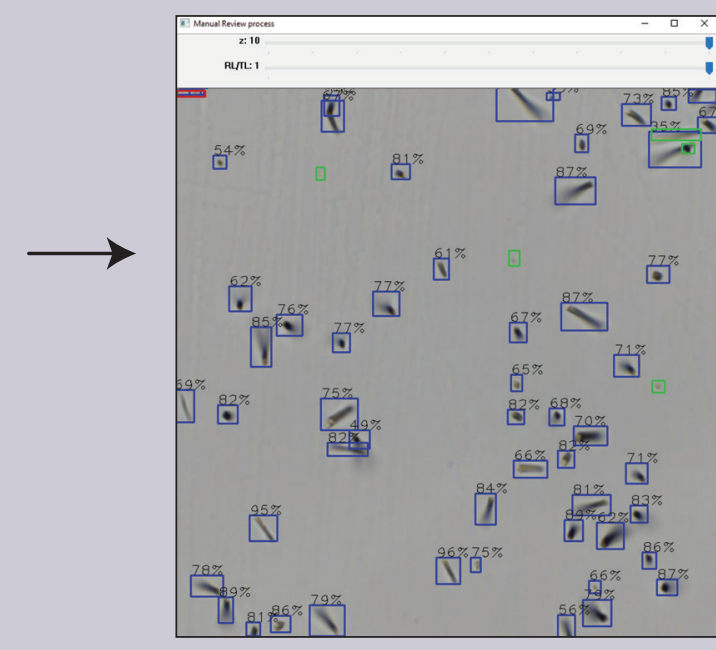

Review the results 